\title{
STOCHASTIC COMPACTNESS AND POINT PROCESSES
}

\author{
L. DE HAAN and S. I. RESNICK
}

(Received 24 November 1981; revised 11 May 1982 and 13 May 1983)

Communicated by R. L. Tweedie

\begin{abstract}
We show that stochastic compactness of partial sums with no normal limit distribution corresponds to stochastic compactness of the point processes generated by the observations so that there exist joint limit distributions for the sample sums and the sample maxima.
\end{abstract}

1980 Mathematics subject classification (Amer. Math. Soc.): primary 60 F 05 ; secondary 62 G 30.

\section{Introduction}

Suppose $\left\{X_{n}, n \geqslant 1\right\}$ are independent, identically distributed random variables with common distribution $F$. Set $S_{n}=\sum_{i=1}^{n} X_{i}, M_{n}=V_{i=1}^{n} X_{i}$. Call $\left\{S_{n}\right\}$ stochastically compact if there exist constants $a_{n} \rightarrow \infty, b_{n} \in R$ such that for every subsequence $\left\{n^{\prime \prime}\right\}$ there exists a further subsequence $\left\{n^{\prime}\right\}$ such that $a_{n^{\prime}}^{-1} S_{n^{\prime}}-b_{n^{\prime}}$ converges weakly to a non-degenerate limit random variable. Similarly, $\left\{M_{n}\right\}$ is stochastically compact if there exist $a_{n} \rightarrow \infty$ such that for every subsequence $\left\{n^{\prime \prime}\right\}$ there is $\left\{n^{\prime}\right\} \subset\left\{n^{\prime \prime}\right\}$ and $a_{n^{\prime}}^{-1} M_{n^{\prime}}$ converges to a proper limit random variable whose distribution is not concentrated at zero. Conditions for stochastic compactness of sums and maxima have been given by Feller (1966) and de Haan and Ridder (1979) (especially Section 2.2) respectively. See also Maller (1981) and Simons and Stout (1978).

We shall show that the two theories of stochastic compactness have a common basis, namely stochastic compactness of point processes, if for the sums we

The work of the second author was partially supported by the Erasmus University, Rotterdam and by NSF Grant MCS-8202335-02. The hospitality of the Econometric Institute, Erasmus University during first semester 1981 is warmly appreciated.

(C) 1984 Australian Mathematical Society $0263-6115 / 84 \$ A 2.00+0.00$ 
exclude the normal distribution from the set of limit distributions. This exclusion makes sense because when sums converge to normality the jumps in the summation process disappear and a limiting point process is trivial and non-informative.

Since asymptotic behavior of sums depends on behavior of both tails of $F$ while corresponding results for maxima depend only on the right tail, the comparison between the two theories is clearest when all random variables $\left\{X_{n}\right\}$ are positive which we henceforth assume.

We now review some notation and definitions connected with weak convergence and point processes. The theory of weak convergence of probability measures is standard and we follow Billingsley (1968) except that " $\Rightarrow$ " denotes weak convergence and we sometimes set weak convergence in the space $D[0, \infty)$ of right continuous functions on $[0, \infty)$ with finite left limits existing on $(0, \infty)$; see Lindvall (1973), Whitt (1980).

Let $E$ be a Euclidean space and suppose $\mathcal{E}$ is the $\sigma$-algebra generated by open sets. All measures on $E$ are assumed to be Radon. Vague convergence of measures $\mu_{n}$ to $\mu$ (written $\mu_{n} \rightarrow^{0} \mu$ ) means

$$
\int_{E} f d \mu_{n} \rightarrow \int_{E} f d \mu
$$

for all continuous, non-negative functions $f$ with compact support. For $x \in E$, $A \in \mathcal{E}$ define the measure $\varepsilon_{x}$ on $E$ by $\varepsilon_{x}(A)=1$ if $x \in A ;=0$ if $x \notin A$. A point measure on $\mathcal{E}$ is a measure of the form $\Sigma_{i} \varepsilon_{x_{j}}$ where $x_{i} \in E$. The set of such point measures is denoted by $M_{p}(E)$. The space $M_{p}(E)$ can be metrized to give the vague topology. See, for example, Neveu (1976) for details.

Although all our random variables are real and finite, it is convenient to regard their state space as $(0, \infty)$ with metric

$$
d^{*}(x, y)=\left|x^{-1}-y^{-1}\right| .
$$

This interchanges the roles of 0 and $\infty$ and makes neighborhoods of $\infty$ bounded. The reason for this convention is that if $a_{n}>0$ and $\nu$ is a measure on $(0, \infty]$ then the statement

$$
n F\left(a_{n} \cdot\right) \stackrel{v}{\rightarrow} \nu(\cdot)
$$

includes the information

$$
n\left(1-F\left(a_{n} x\right)\right) \rightarrow \nu(x, \infty],
$$

at all continuity points $x>0$.

We will need point processes on the space $[0, \infty) \times(0, \infty]$ and it is convenient to metrize this set as follows: For $t_{i} \in[0, \infty), x_{i} \in(0, \infty], i=1,2$ define

$$
d\left(\left(t_{1}, x_{1}\right),\left(t_{2}, x_{2}\right)\right)=\left|t_{1}-t_{2}\right| \vee\left|x_{1}^{-1}-x_{2}^{-1}\right| \text {. }
$$




\section{Stochastic compactness}

The connections between stochastic compactness of point processes, sums and maxima is given in the following theorem. Set $\bar{F}=1-F$.

THEOREM. The following are equivalent

(1)

$$
-2<\lim _{x \rightarrow \infty} \frac{\liminf _{t \rightarrow \infty} \log (\bar{F}(t x) / \bar{F}(t))}{\log x} \leqslant \lim _{x \rightarrow \infty} \frac{\limsup _{t \rightarrow \infty} \log (\bar{F}(t x) / \bar{F}(t))}{\log x}<0 .
$$

(2) There exist constants $a_{n} \rightarrow \infty$ such that $\left\{n F\left(a_{n} \cdot\right)\right\}$ is vaguely relatively compact with the following specification: Every sequence $\left\{n^{\prime \prime}\right\}$ contains a further subsequence $\left\{n^{\prime}\right\}$ such that $\left\{n^{\prime} F\left(a_{n^{\prime}} \cdot\right)\right\}$ is vaguely convergent and if $\nu$ is one of the vague limits then $\nu(\{\infty\})=0$ and $\liminf _{x \rightarrow \infty} \log \nu(x, \infty) / \log x>-2$.

(3) There exist constants $a_{n} \rightarrow \infty$ such that the sequence of point processes

$$
\left\{N_{n}(\cdot)\right\}=\left\{\sum_{k} \varepsilon_{\left(k / n, a_{n}^{-1} X_{k}\right)}\right\}
$$

is relatively compact in the vague topology on $M_{p}([0, \infty) \times(0, \infty])$ with the following specification: Every sequence $\left\{n^{\prime \prime}\right\}$ contains a subsequence $\left\{n^{\prime}\right\}$ such that $\left\{N_{n^{\prime}}\right\}$ converges to a Poisson process on $[0, \infty) \times(0, \infty]$ with mean measure $d t \times d v$ and $\nu(\{\infty\})=0$ and $\lim \inf _{x \rightarrow \infty} \log \nu(x, \infty) / \log x>-2$.

(4) There exist $a_{n} \rightarrow \infty, b_{n} \in R$ such that

$$
\left(a_{n}^{-1} S_{[n \cdot]}-(\cdot) b_{n}, a_{n}^{-1} M_{[n \cdot]}\right)
$$

is relatively compact in $D[0, \infty) \times D[0, \infty)$ and every subsequential limit of $\left\{a_{n}^{-1} S_{[n \cdot]}-(\cdot) b_{n}\right\}$ is a non-terminating Lévy process with no Brownian Motion component.

(5) $\left\{S_{n}\right\}$ is stochastically compact and $F$ is not in the domain of partial attraction of the normal distribution.

(6) $\left\{M_{n}\right\}$ is stochastically compact and any subsequential limit distribution $G$ satisfies $\lim$ inf $_{x \rightarrow \infty} \log \bar{G}(x) / \log x>-2$.

REMARKS. (i) The limits exist in (1) because of subadditivity (Goldie 1977, Matuszewska 1962, Hille 1948).

(ii) The constants $a_{n}$ can always be taken to be $\left.\overleftarrow{F(1}-n^{-1}\right)$ where $\overleftarrow{F}$ is (for example) the right continuous inverse of $F$.

(iii) Of course it is always true that a sequence of probability measures on a product space is relatively compact iff the two marginal sequences of measures are each relatively compact (Billingsley, 1968, page 41 ). The proof of the theorem 
shows that in (4) a stronger statement is possible: One marginal sequence converges along a subsequence if and only if the other marginal sequence converges along the same subsequence. In fact, if (1) holds then

$$
n^{\prime} F\left(a_{n^{\prime}} \cdot\right) \stackrel{v}{\rightarrow} \nu(\cdot)
$$

if and only if convergence along subsequence $n^{\prime}$ holds in (3), (4), (5), (6).

ProOF. We begin by showing (1) is equivalent to the following statement:

(7) There exist $a_{n} \rightarrow \infty$ such that

$$
-2<\lim _{x \rightarrow \infty} \frac{\log \liminf _{n \rightarrow \infty} n \bar{F}\left(a_{n} x\right)}{\log x} \leqslant \lim _{x \rightarrow \infty} \frac{\log \limsup n \bar{F}\left(a_{n} x\right)}{\log x}<0 .
$$

Given (1) we set $a_{n}=\overleftarrow{F(}\left(1-n^{-1}\right)$ so that $\bar{F}\left(a_{n}(1+\varepsilon)\right) \leqslant n^{-1} \leqslant \bar{F}\left(a_{n}(1-\varepsilon)\right)$. Then

$$
\begin{aligned}
\lim _{x \rightarrow \infty} \frac{\log \limsup _{n \rightarrow \infty} n \bar{F}\left(a_{n} x\right)}{\log x} & \leqslant \lim _{x \rightarrow \infty} \frac{\log \limsup _{n \rightarrow \infty} \frac{\bar{F}\left(a_{n}(1+\varepsilon) x(1+\varepsilon)^{-1}\right)}{\bar{F}\left(a_{n}(1+\varepsilon)\right)}}{\log x} \\
& \leqslant \lim _{x \rightarrow \infty} \frac{\log \limsup _{t \rightarrow \infty} \frac{\bar{F}(t x)}{\bar{F}(t)}}{\log x}<0,
\end{aligned}
$$

and a similar argument holds for lim inf so that (7) ensues. (Note, this argument allows the continuity requirement to be dropped in de Haan and Ridder, 1979.) Conversely, if (7) is true then there is an $x_{1}$ such that $\liminf _{n \rightarrow \infty} n \bar{F}\left(a_{n} x_{1}\right) \geqslant$ $x_{1}^{-2+\delta}>0$ for some $\delta \in(0,2)$ and so there exists $c>0$ such that for $n$ large $n \bar{F}\left(a_{n} x_{1}\right) \geqslant c$. For $t>0$ define $n(t)=\inf \left\{n: a_{n+1}>t\right\}$ so that $a_{n(t)} \leqslant t<a_{n(t)+1}$ and the following inequality is clear:

$$
\frac{\bar{F}\left(t x_{1} \cdot x x_{1}^{-1}\right)}{\bar{F}\left(t x_{1}\right)} \leqslant \frac{n(t) \bar{F}\left(a_{n(t)} x\right)}{(n(t)+1) \bar{F}\left(a_{n(t)+1} x_{1}\right)} \cdot \frac{n(t)+1}{n(t)} .
$$

Therefore

$$
\lim _{x \rightarrow \infty} \frac{\log \limsup _{t \rightarrow \infty} \bar{F}(t x) / \bar{F}(t)}{\log x} \leqslant \lim _{x \rightarrow \infty} \frac{\log \limsup _{n \rightarrow \infty} n \bar{F}\left(a_{n} x\right)}{\log x}<0,
$$

and now (1) is evident.

Next we verify the equivalence of (1) and (2). Supposing (1) true we get from (7) that for sufficiently large $x, \lim \sup _{n \rightarrow \infty} n \bar{F}\left(a_{n} x\right) \leqslant x^{-\rho}$ for some $\rho \in(0,2)$. For small $x$ the liminf inequality in (1) gives after a change of variable $\lim \sup _{t \rightarrow \infty} \bar{F}(t x) / \bar{F}(t) \leqslant x^{-2+\delta}$ for $\delta \in(0,2)$ and as above this leads to 
$\lim \sup _{n \rightarrow \infty} n \bar{F}\left(a_{n} x\right) \leqslant c x^{-2+\delta}$ for small $x$. The selection theorem then applies to give $\left\{n F\left(a_{n} \cdot\right)\right\}$ vaguely relatively compact. Furthermore, if $v$ is a vague limit we have for some subsequence $\left\{n^{\prime}\right\}$ that $n^{\prime} \bar{F}\left(a_{n^{\prime}} x\right) \rightarrow \nu(x, \infty]$ at continuity points $x$. So we have on the one hand

$$
\nu(\{\infty\})=\lim _{x \rightarrow \infty} \nu(x, \infty]=\lim _{x \rightarrow \infty} \lim _{n^{\prime} \rightarrow \infty} n^{\prime} \bar{F}\left(a_{n^{\prime}} x\right) \leqslant \lim _{x \rightarrow \infty} \limsup _{n \rightarrow \infty} n \bar{F}\left(a_{n} x\right)=0,
$$

and on the other

$$
\begin{aligned}
\liminf _{x \rightarrow \infty} \log \nu(x, \infty) / \log x & =\liminf _{x \rightarrow \infty} \log \left(\lim _{n^{\prime} \rightarrow \infty} n^{\prime} \bar{F}\left(a_{n^{\prime}} x\right)\right) / \log x \\
& \geqslant \liminf _{x \rightarrow \infty} \log \left(\liminf _{n \rightarrow \infty} n \bar{F}\left(a_{n} x\right)\right) / \log x>-2 .
\end{aligned}
$$

Conversely, suppose (2) is true. Towards the goal of doing a proof by contradiction note the following string of equivalences:

$$
\begin{gathered}
\lim _{x \rightarrow \infty} \frac{\log \limsup _{n \rightarrow \infty} n \bar{F}\left(a_{n} x\right)}{\log x}<0 \\
\Leftrightarrow \quad \lim _{x \rightarrow \infty} \limsup _{n \rightarrow \infty} n \bar{F}\left(a_{n} x\right)=0 \quad \text { (see de Haan and Ridder, 1979) } \\
\Leftrightarrow \quad \lim _{x \rightarrow \infty} n \bar{F}\left(a_{n} x\right)=0 \quad \text { uniformly in } n \\
\Leftrightarrow \quad \text { for any integer valued function } n(x) \\
\quad \lim _{x \rightarrow \infty} n(x) \bar{F}\left(a_{n(x)} x\right)=0 .
\end{gathered}
$$

So if the right-most inequality in (7) fails there exists an integer valued function $\eta(x)$ and $c>0$ such that

$$
\limsup _{x \rightarrow \infty} n(x) \bar{F}\left(a_{n(x)} x\right)=c>0 .
$$

Then we can find $\left\{x_{k}\right\}$ such that $n\left(x_{k}\right) \rightarrow \infty$ and $n\left(x_{k}\right) \bar{F}\left(a_{n\left(x_{k}\right)} x_{k}\right) \geqslant \frac{1}{2} c$. From relative compactness there exists a subsequence $\left\{n^{\prime}\right\} \subset\left\{n\left(x_{k}\right)\right\}$ and a measure $\nu$ with $v(\{\infty\})=0$ and

$$
n^{\prime} F\left(a_{n^{\prime}} \cdot \stackrel{\bullet}{\rightarrow} \nu .\right.
$$

Then for any $x$ which is not an atom of $\nu$ we have

$$
\nu(x, \infty]=\lim _{n^{\prime} \rightarrow \infty} n^{\prime} \bar{F}\left(a_{n^{\prime}} x\right) \geqslant \liminf _{k \rightarrow \infty} n\left(x_{k}\right) \bar{F}\left(a_{n\left(x_{k}\right)} x_{k}\right) \geqslant \frac{1}{2} c .
$$

Since $x$ can be arbitrarily large we get $\nu(\{\infty\}) \geqslant \frac{1}{2} c$ which is a contradiction. Thus (2) implies the lim sup inequality of (7). The lim inf inequality in (7) follows :rom the following lemma supplied by Dr. A. A. Balkema. The proof is omitted. jee Maller (1980) where similar methods are employed. 
LEMMA. Suppose $f$ is non-decreasing. If

$$
\lim _{x \rightarrow \infty} \limsup _{t \rightarrow \infty} \frac{f(t+x)-f(t)}{x}=c>0,
$$

then there is a sequence $t_{n} \rightarrow \infty$ and a function $\varphi(x) \geqslant c x$ such that

$$
f\left(t_{n}+x\right)-f\left(t_{n}\right) \rightarrow \varphi(x) \quad(n \rightarrow \infty)
$$

at all $x$ which are continuity points of $\varphi$.

We now consider the equivalence of (2) and (3) making use of the fact that a sequence of point processes converges weakly if and only if corresponding Laplace functionals converge (Neveu, 1976).

The details are sketched as follows: Let $f:[0, \infty) \times(0, \infty] \rightarrow[0, \infty)$ be a continuous function with compact support and set

$$
\lambda_{n}(d s, d x)=\sum_{k} \varepsilon_{k n^{-1}}(d s) P\left[a_{n}^{-1} X_{1} \in d x\right],
$$

so that for some subsequence $\left\{\boldsymbol{n}^{\prime}\right\}$

$$
\lambda_{n^{\prime}}(d s, d x) \stackrel{0}{\rightarrow} d s \times \nu(d x) \text { on }[0, \infty) \times(0, \infty]
$$

if and only if $n^{\prime} F\left(a_{n^{\prime}} \cdot \stackrel{v}{\rightarrow} \nu(\cdot)\right.$ as $n^{\prime} \rightarrow \infty$. Therefore

$$
\begin{aligned}
\sum_{k=1}^{\infty} \int_{(0, \infty]} & \left(1-e^{-f\left(k n^{-1}, x\right)}\right) P\left[a_{n} X_{1} \in d x\right] \\
= & \iint_{[0, \infty) \times(0, \infty]}\left(1-e^{-f}\right) d \lambda_{n} \rightarrow \iiint_{[0, \infty) \times(0, \infty]}\left(1-e^{-f(s, x)}\right) d s \nu(d x),
\end{aligned}
$$

along $\left\{n^{\prime}\right\}$ if and only if $n^{\prime} F\left(a_{n^{\prime}} \cdot \stackrel{\mathcal{D}}{\rightarrow} \nu\right.$. Finally

$$
\begin{aligned}
E \exp \{- & \left.\iint_{[0, \infty) \times(0, \infty)} f(s, x) N_{n}(d s, d x)\right\} \\
& =E \exp \left\{-\sum_{k} f\left(k n^{-1}, a_{n}^{-1} X_{k}\right)\right\} \\
& =\prod_{k}\left(1-\int_{(0, \infty]}\left(1-e^{-f\left(k n^{-1}, x\right)}\right) P\left[a_{n}^{-1} X_{1} \in d x\right]\right) \\
& =\exp \left\{\sum_{k} \log \left(1-\int\left(1-e^{-f\left(k n^{-1}, x\right)}\right) P\left[a_{n}^{-1} X_{1} \in d x\right]\right)\right\} \\
& \approx \exp \left\{-\sum_{k} \int\left(1-e^{-f\left(k n^{-1}, x\right)}\right) P\left[a_{n}^{-1} X_{1} \in d x\right]\right\} \\
& \rightarrow \exp \left\{-\iint_{[0, \infty) \times(0, \infty]}\left(1-e^{-f(s, x)}\right) d s \nu(d x)\right\},
\end{aligned}
$$


along $\left\{n^{\prime}\right\}$ if and only if $n^{\prime} F\left(a_{n^{\prime}} \cdot\right) \rightarrow^{0} \nu$. Since

$$
\exp \left\{-\int_{[0, \infty) \times(0, \infty]}\left(1-e^{-f(s, x)} d s \nu(d x)\right)\right\}
$$

is the Laplace functional of a Poisson process with mean measure $d s \times d \nu$ we have established the equivalence of (1), (2) and (3).

Next we show (3) implies (4). See Durrett and Resnick (1978), Resnick and Greenwood (1979). Suppose $\left\{n^{\prime}\right\}$ is a sequence of integers such that $n^{\prime} F\left(a_{n^{\prime}} \cdot\right)$ $\rightarrow^{v} \nu$ so that along this subsequence $N_{n^{\prime}}$ converges to a limit Poisson process $N$ with mean measure $d t \times d \nu$. We represent $N$ as $\Sigma_{k} \varepsilon_{\left(t_{k}, j_{k}\right)}$. We first check that $\nu$ is a Lévy measure; that is, $\int_{(0, \infty)}\left(x^{2} \wedge 1\right) \nu(d x)<\infty$. From (1) there exists $x_{0}$ and $\delta \in(0,2)$ such that $\liminf _{t \rightarrow \infty} \bar{F}(t x) / \bar{F}(t)>x^{-2+\delta}$ for $x \geqslant x_{0}$. Changing variables $y=x^{-1}$ and taking reciprocals gives $\lim \sup _{t \rightarrow \infty} \bar{F}(t y) / \bar{F}(t) \leqslant y^{-2+\delta}, y \leqslant x_{0}^{-1}$ and so $\nu(y, \infty) \leqslant y^{-2+\delta}, y \leqslant x_{0}^{-1}$. Therefore

$$
\int_{\left(0, x_{0}^{-1}\right)} y \nu(y, \infty) d y \leqslant \int_{\left(0, x_{0}^{-1}\right)} y^{-1+\delta} d y<\infty,
$$

and this confirms that $\nu$ is a Lévy measure.

Now we use $N_{n^{\prime}} \Rightarrow N$ and the continuous mapping theorem (Billingsley, 1968) to get $\left(n^{\prime} \rightarrow \infty\right)$

$$
\begin{aligned}
Z_{n^{\prime}, \varepsilon}(\cdot) & :=\sum_{i=1}^{\left[n^{\prime} \cdot\right]} a_{n^{\prime}}^{-1} X_{i} 1_{\left[a_{n}, X_{i}>\varepsilon\right]}-\left[n^{\prime} \cdot\right] E a_{n^{\prime}}^{-1} X_{i} 1_{\left[a_{n}^{-1} X_{i} \in(\varepsilon, 1]\right]} \\
& \Rightarrow Z_{\varepsilon}(\cdot)=\sum_{t_{i} \leqslant(\cdot)} j_{i} 1_{\left[j_{i}>\varepsilon\right]}-(\cdot) \int_{(\varepsilon, 1]} u \nu(d u)
\end{aligned}
$$

on $D[0, \infty)$ for $\varepsilon, 1$ assumed continuity points of $\nu(x, \infty)$. Because $\nu$ is a Lévy measure, as $\varepsilon \downarrow 0 \quad Z_{\varepsilon}(t)$ converges a.s. and locally uniformly in $t$ to a Lévy process $Z$ with Lévy measure $\nu$ (Ito, 1969). Set

$$
Z_{n^{\prime}}(t)=\sum_{i=1}^{\left[n^{\prime} t\right]} a_{n^{\prime}} X_{i}-\left[t n^{\prime}\right] E a_{n^{\prime}} X_{1} 1_{\left[a_{n}, X_{1} \leq 1\right]} .
$$

Then $Z_{n^{\prime}} \Rightarrow Z$ in $D[0, \infty)$ will follow from the above facts and Billingsley, 1968, Theorem 4.2, p. 25 provided we show for any $\eta>0$

$$
\lim _{\varepsilon \downarrow 0} \limsup _{n \rightarrow \infty} P\left[\sup _{0 \leqslant t \leqslant 1}\left|Z_{n^{\prime}}(t)-Z_{n^{\prime}, \varepsilon}(t)\right|>\eta\right]=0 .
$$

This probability is

$$
P\left[\sup _{0<i<1}\left|\sum_{i=1}^{\left[n^{\prime} t\right]} a_{n^{\prime}}^{-1} X_{i} 1_{\left[a_{n}^{-1} X_{i}<\varepsilon\right]}-\left[n^{\prime} t\right] E a_{n^{\prime}}^{-1} X_{i} 1_{\left[a_{n}^{-1} X_{1}<\varepsilon\right]}\right|>\eta\right],
$$


and applying Kolmogorov's inequality we get the upper bound

$$
\eta^{-2} n^{\prime} \operatorname{Var}\left(a_{n^{\prime}}^{-1} X_{1} 1_{\left[a_{n}^{-1} X_{1} \leqslant \varepsilon\right]}\right) \leqslant \eta^{-2} a_{n^{\prime}}^{-2} n^{\prime} V\left(\varepsilon a_{n^{\prime}}\right),
$$

where as usual $V(x)=E X_{1}^{2} 1_{\left[X_{1} \leqslant x\right]}$. However

$$
\lim _{\varepsilon \downarrow 0} \limsup _{n \rightarrow \infty} a_{n}^{-2} n V\left(\varepsilon a_{n}\right) \leqslant \lim _{\varepsilon \downarrow 0} \limsup _{n \rightarrow \infty} \int_{0}^{\varepsilon} 2 u n \bar{F}\left(a_{n} u\right) d u .
$$

By an argument similar to the one which proved $v$ is a Lévy measure we get for small enough $u$ that $\lim \sup _{n \rightarrow \infty} n F\left(a_{n} u\right) \leqslant c u^{-2+\delta}$ and so (8) is bounded above by

$$
\lim _{\varepsilon \downarrow 0}(\text { const }) \int_{0}^{\varepsilon} u^{-1+\delta} d u=0
$$

as required.

We thus obtain

$$
Z_{n^{\prime}}(\cdot)=a_{n^{\prime}}^{-1} S_{\left[n^{\prime} \cdot\right]}-(\cdot) b_{n^{\prime}} \Rightarrow Z,
$$

and $Z$ is a Lévy process with no Brownian Motion component. To get the asserted joint convergence in (4) apply the functional $T: D[0, \infty) \rightarrow D[0, \infty) \times$ $D[0, \infty)$ defined by

$$
(T x)(t)=\left(x(t), \sup _{0<s \leqslant t}(x(s)-x(s-))\right)
$$

to (9) and use the continuous mapping theorem (Billingsley, 1968).

The implication (4) $\rightarrow(5)$ is easy to check because one can use the evaluation map $(x, y) \rightarrow x(1)$ (from $D[0, \infty) \times D[0, \infty) \rightarrow R$ ) to get $\left\{S_{n}\right\}$ is stochastically compact and Theorem 3 of Maller (1980) to check $F$ is not in the domain of partial attraction of the normal (since it is not in the domain of partial attractior of an infinitely divisible distribution with nonzero normal component).

The implication $(5) \rightarrow(1)$ follows from the work of Simons and Stout $(1978, p$ 298) and Maller (1981, (1.5)-(1.8)).

Next (3) implies (6) because from (3) we apply the functional $\xi: M_{p}([0, \infty) \times$ $(0, \infty]) \rightarrow R$ defined by

$$
\xi N_{n}=\sup \left\{a_{n}^{-1} X_{k}: k n^{-1} \leqslant 1\right\}=M_{n} / a_{n},
$$

and (6) follows. From (6) we get

$$
P\left[a_{n^{\prime}}^{-1} M_{n^{\prime}} \leqslant x\right] \rightarrow G(x),
$$

as $n^{\prime} \rightarrow \infty$ if and only if $F^{n^{\prime}}\left(a_{n^{\prime}} x\right) \rightarrow G(x)$. This is equivalent to $n^{\prime} \bar{F}\left(a_{n^{\prime}} \cdot\right) \rightarrow \nu(\cdot$ where $\nu(x, \infty)=-\log G(x)$, and (2) is evident. The proof is complete. 
ReMarks . (iv) From the proof it follows that if the sequence of maxima is stochastically compact then $\liminf _{n \rightarrow \infty} F^{n}\left(a_{n} x\right) \geqslant \exp \left\{-c_{1} x^{-\rho}\right\}$, some extremevalue distribution, for $x \geqslant 1$ and $\lim _{\sup _{n \rightarrow \infty}} F^{n}\left(a_{n} x\right) \leqslant \exp \left\{-c_{2} x^{-\rho}\right\}$ for $x \leqslant 1$, if we choose $a_{n}=\overleftarrow{F(}(1-1 / n)$. Compare with the bounds obtained in Anderson (1970).

(v) The Definition 2.1.2 in de Haan and Ridder (1979) of stochastic compactness precludes any limit distribution from having mass at zero. However, in order to get the set of equivalent statements for stochastic compactness of sample maxima in de Haan and Ridder (1979, Theorem 2.2.1) it is sufficient to require that any limit distribution be proper and is not concentrated at zero. The latter condition implies that for any subsequence $n^{\prime}$ along which convergence takes place, $\lim _{n^{\prime} \rightarrow \infty} n^{\prime}\left\{1-F\left(a_{n^{\prime}} x\right)\right\}>0$ for some $x>0$. Line -1 , page 292 of de Haan and Ridder (1979) shows we should check $\liminf _{n \rightarrow \infty} n\left(1-F\left(a_{n} x\right)\right)>0$ for some $x>0$. Suppose this is not true; then for any $r, x_{r}, \varepsilon_{r}$ there exists $n_{r}$ such that $n_{r}\left\{1-F\left(a_{n_{r}} x_{r}\right)\right\}<\varepsilon_{r}$. Take $x_{r} \downarrow 0, \varepsilon_{r} \downarrow 0$ then for all $x>0 n_{r}\left\{1-F\left(a_{n_{r}} x_{r}\right)\right\}$ $\leqslant n_{r}\left\{1-F\left(a_{n_{r}} x_{r}\right)\right\}<\varepsilon_{r}$. Hence $\lim _{r \rightarrow \infty} n_{r}\left\{1-F\left(a_{n_{r}} x\right)\right\}=0$ for all $x>0$, a contradiction.

\section{References}

C. W. Anderson (1970), 'Extreme value theory for a class of discrete distributions with applications to some stochastic process', J. Appl. Probab. 7, 99-113.

P. Billingsley (1968), Convergence of probability measures (Wiley, New York).

R.Durrett and S. Resnick (1978), 'Functional limit theorems for dependent variables', Ann. Probab. 6, 829-846.

W. Feller (1966), 'On regular variation and local limit theorems', Proc. Fifth Berkeley Symp. Math. Statist. Probability 2, 373-388.

C. Goldie (1977), 'Convergence theorems for empirical Lorenz curves and their inverses', Adv. in Appl. Probab. 9, 765-791.

L. de Haan and G. Ridder (1979), 'Stochastic compactness of sample extremes', Ann. Probab. 7, 290-303.

E. Hille and R. S. Phillips (1948), Functional analysis and semigroups (Amer. Math. Soc. Colloq. Publ, vol. 31).

K. Ito (1969), Stochastic processes (Lecture Notes, Series no. 16, Mathematisk Institut, Aarhus University).

T. Lindvall (1973), 'Weak convergence of probability measures and random functions in the function

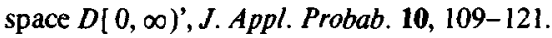

R. A. Maller (1980), 'A note on domains of partial attraction', Ann. Probab. 8, 576-583.

R. A. Maller (1981), 'Some properties of stochastic compactness', J. Austral. Math. Soc. Ser A 30, 263-277.

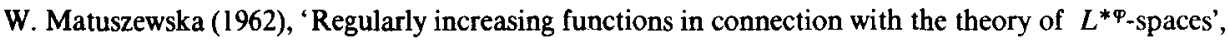
Studia Math. 21, 317-344.

J. Neveu (1976), Processus ponctuels, Ecole d'Eté de Probabilités de Saint-Flour (Lecture Notes in Mathematics 598, Springer, Berlin). 
S. Resnick and P. Greenwood (1979), 'A bivariate stable characterization and domains of attraction', J. Multivariate Anal. 9, 206-221.

G. Simons and W. Stout (1978), 'A weak invariance principle with applications to domains of attraction', Ann. Probab. 6, 294-315.

W. Whitt (1980), 'Some useful functions for functional limit theorems', Math. Oper. Res. 5, 67-85.

Econometric Institute

Erasmus University

Rotterdam

Holland

\author{
Department of Statistics \\ Colorado State University \\ Fort Collins, Colorado 80523 \\ U.S.A.
}

